\title{
On the Stability of a Double Integration Delta Modulator
}

\author{
Nielsen, Palle Tolstrup
}

Published in:

I E E E Transactions on Communications

Link to article, DOI:

10.1109/TCOM.1971.1090641

Publication date:

1971

Document Version

Publisher's PDF, also known as Version of record

Link back to DTU Orbit

Citation (APA):

Nielsen, P. T. (1971). On the Stability of a Double Integration Delta Modulator. I E E E Transactions on Communications, 19(3), 364-366. https://doi.org/10.1109/TCOM.1971.1090641

\section{General rights}

Copyright and moral rights for the publications made accessible in the public portal are retained by the authors and/or other copyright owners and it is a condition of accessing publications that users recognise and abide by the legal requirements associated with these rights.

- Users may download and print one copy of any publication from the public portal for the purpose of private study or research.

- You may not further distribute the material or use it for any profit-making activity or commercial gain

- You may freely distribute the URL identifying the publication in the public portal

If you believe that this document breaches copyright please contact us providing details, and we will remove access to the work immediately and investigate your claim. 
Fig. 3 is a simplified block diagram. The input signal voltage is commutated to the storage condensers by successive closures of the input switches at a rate set by oscillator 1, and commutated thence to the output terminal by successive elosures of the output switches at a slower rate under control of oscillator 2. The latter runs at a fixed rate of $16000 \mathrm{~Hz}$, a frequency great enough to avoid generation of audible components due to commutation. Oscillator 1 has a higher frequency which is adjustable. The frequency demultiplication ratio is equal to the ratio of the oscillator frequencies; for example, it is 50 percent with oscillator 1 set at $32000 \mathrm{~Hz}$.

Both switch control circuits begin each reset cycle simultaneously with the first input and output switches closed. Then the switch closures progress to the right in Fig. 3, the input rate being greater than the output. With no glottal synchronizing signal present, the input circuit reaches its last position and waits there until the output circuit reaches its last switch. Then the next pulse from oscillator 2 resets both circuits and the cycle repeats. If a glottal synchronizing signal is received, the circuits are reset immediately regardless of how far the current storage--readout cycle has progressed.

Evaluation of this unscrambler was performed with taped helium speech stimuli, as well as in on-line operation during dives. Highly successful performance was demonstrated with divers at pressure depths as great as $1000 \mathrm{ft}$. Objective word intelligibility test results were 88 percent at $500 \mathrm{ft}$ and 78 percent at $800 \mathrm{ft}$, as compared with previously reported intelligibility of 5 percent for uncorrected helium speech at a depth of $600 \mathrm{ft}$.

\section{ACKNOWLEDGMENT}

Dive test facilities were provided by Ocean Systems, Inc. The equation relating frequency and depth was derived by Dr. G. R. Gamertsfelder, and the unscrambler was designed by J. W. Gray, both of Singer-General Precision.

\section{REFERENCES}

[1] L. J. Gerstman, G. R. Gamertsfelder, and A. Goldberger, J. Acoust.

[2] Soc. Amer., vol. 40, 1966, p. 1283 (A).

[3] Lieberman et al., Psychol. Monogr., vol. 68, no. 8, 1954, pp. 1-13.

\section{On the Stability of a Double Integration Delta Modulator}

\section{P. TOLSTRUP NIELSEN}

Abstract-A delta modulator with a second-order network in the feedback path is considered. For zero input signal the possible modes of oscillation are determined as a function of the zero of the linear network. The method could be employed with other types of feedback networks. A comparison is made with experimental results.

\section{INTRODUCTION}

It is well known that the performance of a delta modulation system in many cases can be improved by choosing a more complicated network than a single integrator in the feedback path of the modulator. With the configuration in Fig. 1 an of ten suggested transfer function for the linear network is

$$
H(s)=\frac{1+s \tau_{0}}{\left(1+s \tau_{1}\right)\left(1+s \tau_{2}\right)} .
$$

Paper approved by the Wire Communication Committee of the IEEE Communication Technology Group for publication without oral presentation. Manuscript received February 4, 1970.

The author is with the Laboratory for Communication 'Theory Technical University of Denmark, Lyngby, Denmark.

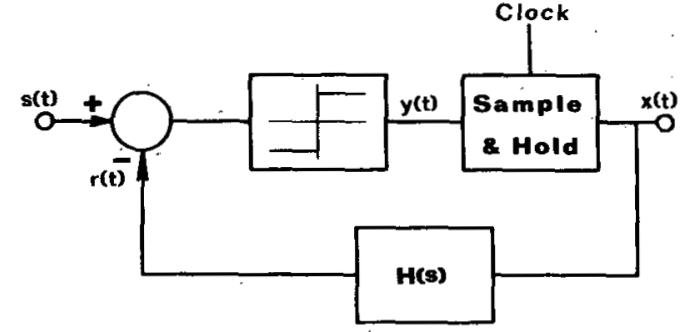

Fig. 1. Delta modulator under consideration.

The choice of the parameters $\tau_{1}$ and $\tau_{2}$ chiefly depends on the spectrum of the transmitted waveform. $s(t)$, whereas the zero is needed because of the demand for stability. From a quantizing noise viewpoint it is desirable to keep $\tau_{0}$ as small as possible. In fact it can be shown that with all other parameters fixed, the ratio of rms signal to rms noise is approximately inversely proportional to $\tau_{0}$. However, if $\tau_{0}$ is decreased below a certain limit, the system becomes unstable, and the follow-up characteristics of the modulator are destroyed. Hence there is an optimum value of $\tau_{0}$ which yields the best overall system performance. The purpose of this paper is to show how the degree of unstability depends on the choice of $\tau_{0}$ when all other parameters remain fixed.

\section{Possible Modes of Oscillation}

Consider the delta modulator in Fig. 1. For the purpose of this evaluation it is assumed that the input signal $s(t)$ is zero and that the $\mathrm{dc}$ balance of the modulator is perfect, i.e., the number of ones equals the number of zeros in the output bit stream. Under these conditions the signal $x(t)$ will be a symmetrical square wave, with a half-period equal to $n T$, where $T$ is the reciprocal of the clock frequency and $n$ is some integer, $n \geq 1$. We denote the oscillation frequency by

$$
f_{0}=\frac{1}{2 n T}=\frac{f_{p}}{2 n}
$$

where $f_{p}$ is the clock frequency. The case $n=1$ corresponds to the highest possible degree of stability. If $n$ is high, the oscillation will be of relatively low frequency and will therefore appear at the output of the demodulator with high amplitude. Our object is to determine the possible values of $n$ for a given value of $\tau_{0}$.

All the waveforms of the system are shown in Fig. 2 for the case of a stationary oscillation. It is readily seen that $y(t)$ is a true replica of $x(t)$, except for a delay $t_{d}$ due to the linear network $H(s)$ and the phase shift of $180^{\circ}$ due to the change of sign at the input of the comparator.

If the oscillation is to continue, the following inequality must be satisfied:

$$
(n-1) T<t_{d}<n T .
$$

Our next objective is to calculate the zero-crossing delay $t_{d}$ for the transfer function (1). It is shown in the Appendix that $t_{d}$ can be found by solving the equation

$$
\begin{array}{r}
1+\frac{2 c_{1}}{1+\exp \left(-n T / \tau_{1}\right)} \exp \left(-\frac{t_{d}}{\tau_{1}}\right)+\frac{2 c_{2}}{1+\exp \left(-n T / \tau_{2}\right)} \\
\cdot \exp \left(-\frac{t_{d}}{\tau_{2}}\right)=0
\end{array}
$$

where $c_{1}$ and $c_{2}$ are given by

$$
\begin{gathered}
c_{1} \triangleq \frac{\tau_{0}-\tau_{1}}{\tau_{1}-\tau_{2}} \\
c_{2} \triangleq \frac{\tau_{0}-\tau_{2}}{\tau_{2}-\tau_{1}} .
\end{gathered}
$$



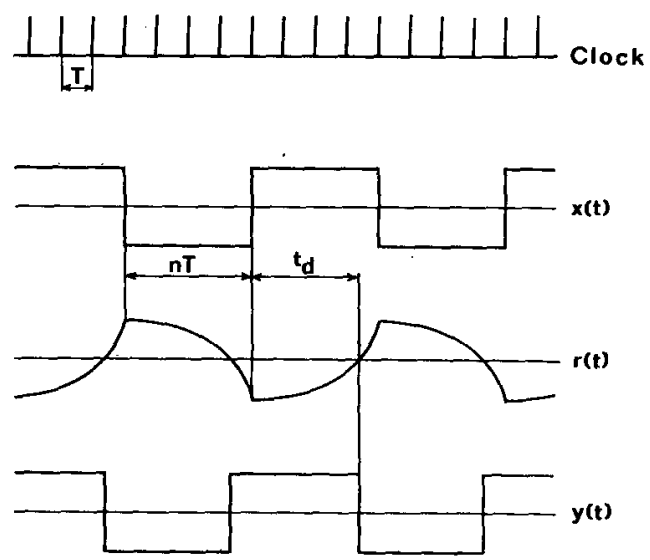

Fig. 2. Waveforms in oscillating system.

If we define

$$
\alpha \triangleq \frac{n T-t_{d}}{T}
$$

the inequality (3) can also be expressed as

$$
0 \leq \alpha<1 \text {. }
$$

The quantity $\alpha$ was calculated by numerical methods from (4) for several values of $n$ and $\tau_{0}$. In this calculation the other parameters were chosen as follows:

$$
\tau_{1}=1200 \mu \mathrm{s}, \quad \tau_{2}=165 \mu \mathrm{s}, \quad T=18 \mu \mathrm{s} .
$$

These are typical values for a delta modulation system designed for telephone quality speech transmission.

The result is shown in Fig. 3, where $\alpha$ is plotted versus $\tau_{0}$ with $n$ as a parameter. Due to the choice of finite values for $\tau_{1}$ and $\tau_{2}$, a lower limit for $f_{0}$ is seen to exist even for $\tau_{0}=0 . n=2$ is a possible mode for high values of $\tau_{0}$. Consequently, no improvement of system performance could be expected when choosing $\tau_{0}>1.5^{\prime} t^{\prime}$.

\section{Experimental Results}

Tests were carried otit in the laboratory in an attempt to establish the modes of oscillation predicted in Fig. 3. It turned out that for $\tau_{0}=T$, the only possible output pattern was

$$
\underline{101010} \cdots
$$

whereas for $\tau_{0}=0.25 T$ three patterns could be stable:

$$
\begin{aligned}
& \underline{101010} \ldots \\
& \underline{11001100 . .} \\
& 111000111000 \cdots .
\end{aligned}
$$

The number of observed modes is considerably less than the theoretically expected number which, from Fig. 3 , in the two cases should be 4 and 8 . Obviously oscillations corresponding to high values of $\alpha$ are hard to establish because of noise and jitter present in the system.

However, if a random signal is applied to the input of the delta modulator, short bursts of these high-order oseillations may occur from time to time, and unless the frequency of oseillation is well out of the voice band, these bursts will have enough inband energy to cause a noticeable decrease in the output SNR.

Experimental work by de Jager [1], Tomozawa and Kaneko [2], and other authors indicates that the optimum choice of $\tau_{0}$ (maximizing the output SNR) is very close to $T$. From Fig. 3 this choice is seen to eliminate oscillations at frequencies lower than about $7 \mathrm{kHz}$ which is in good agreement with the previous discussion.

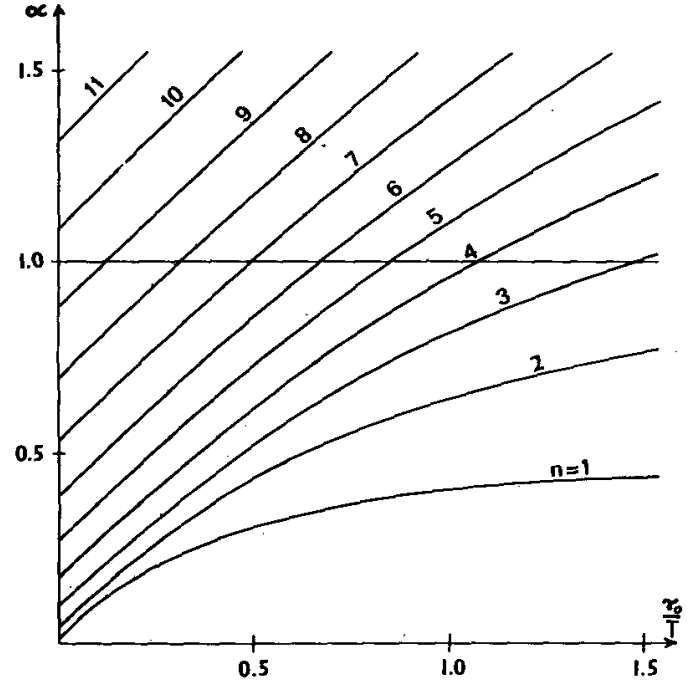

Fig. 3. Stability factor $\alpha=\left(n T-t_{d}\right) / T, \tau_{1}=1200 \mu \mathrm{s}, \tau_{2}=165 \mu \mathrm{s}$, corresponding $\alpha$ satisfies $0<\alpha<1$

\section{CONCLUSION}

For a specific second-order delta modulator the possible modes of oscillation for zero input signal were determined as a function of $\tau_{0}$, the zero of the feedback transfer function. The approach is general in nature and could be employed with other types of transfer functions. Since the analysis presented is valid only under the idle condition, it cannot lead to a rigorous optimization of $\boldsymbol{r}_{0}$. Nevertheless it should be clear that some relation exists between the degree of stability in the idle case and the follow-up characteristics when a signal is applied. The observation by other authors that a value of $\tau_{0}$ close to $T$ seems to be an optimal choice, is in good agreement with the results presented here.

\section{APPENDIX}

Let $u(t)$ be the function defined in Fig. 4. Then the Laplace transform of $u(t)$ is

$$
U(s)=\frac{1}{s} \frac{1-\exp (-a s)}{1+\exp (-a s)} .
$$

This signal is applied to the input of $H(s)$. The transform of the output is

$$
V(s)=\frac{H(s)}{s} \frac{1-\exp (-a s)}{1+\exp (-a s)} .
$$

From the theory of Laplace transforms we know that if

$$
G(s)=\mathfrak{L}\{g(t)\}
$$

where $\mathscr{L}\{\cdot\}$ denotes Laplace transform, then

$G(s) \frac{1-\exp (-a s)}{1+\exp (-a s)}=\mathscr{L}\left\{-g(t)+2 \sum_{0 \leq q<t / a} \exp (j \pi q) g(t-a q)\right\}$

where $q=0,1,2, \cdots$ and $a$ is a real constant, $a>0$.

To invoke (13) we set

$$
G(s)=\frac{H(s)}{s}=\frac{1+s \tau_{0}}{s\left(1+s \tau_{1}\right)\left(1+s \tau_{2}\right)}
$$

and

$$
g(t)=1+c_{1} \exp \left(\frac{t}{\tau_{1}}\right)+c_{2} \exp \left(\frac{t}{\tau_{2}}\right)
$$


with

$$
c_{1}=\frac{\tau_{0}-\tau_{1}}{\tau_{1}-\tau_{2}}
$$

and

$$
c_{2}=\frac{\tau_{0}-\tau_{2}}{\tau_{2}-\tau_{1}} .
$$

From (11) and (13) we obtain

$$
\begin{aligned}
v(t)= & -\left[1+c_{1} \exp \left(-\frac{t}{\tau_{1}}\right)+c_{2} \exp \left(-\frac{t}{\tau_{2}}\right)\right] \\
& +2 \sum_{0 \leq q<t / a} \exp (j \pi q)\left[1+c_{1} \exp \left(-\frac{t-a q}{\tau_{1}}\right)\right. \\
& \left.+c_{2} \exp \left(-\frac{t-a q}{\tau_{2}}\right)\right] .
\end{aligned}
$$

Only the stationary solution is of interest, so we look for an expression for $v(t)$ in the interval

$$
a m<t \leq a(m+1)
$$

where $m$ is a positive integer. We define

$$
t^{\prime}=t-a m
$$

and obtain for the stationary solution in the interval (19)

$$
\begin{aligned}
r\left(t^{\prime}\right)= & \lim _{m \rightarrow \infty} v\left(t^{\prime}\right) \\
= & \lim _{m \rightarrow \infty}\left\{2 \sum _ { \mathrm { q } = 0 } ^ { m } \operatorname { e x p } ( j \pi q ) \left[1+c_{1} \exp \left(-\frac{t^{\prime}+a m-a q}{\tau_{1}}\right)\right.\right. \\
& \left.+c_{2} \exp \left(-\frac{t^{\prime}+a m-a q}{\tau_{2}}\right)\right] \\
& \left.-\left[1+c_{1} \exp \left(-\frac{t^{\prime}+a m}{\tau_{1}}\right)+c_{2} \exp \left(-\frac{t^{\prime}+a m}{\tau_{2}}\right)\right]\right\} .
\end{aligned}
$$

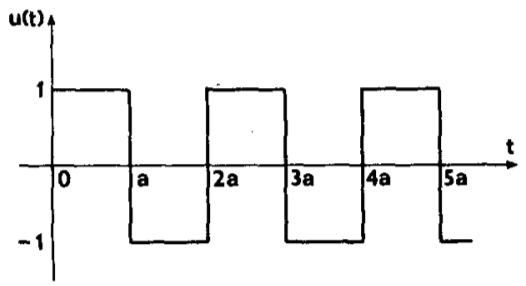

Fig. 4. Definition of function $u(t)$.

But

$$
\sum_{q=0}^{m} \exp (j \pi q) \exp \left(\frac{a q}{\tau}\right)=\frac{1-(-\exp (a / \tau))^{m+1}}{1+\exp (a / \tau)} .
$$

Substituting (22) in (21) we get in the limit

$$
\begin{aligned}
r\left(t^{\prime}\right)=(-1)^{m} & {\left[1+\frac{2 c_{1}}{1+\exp \left(-a / \tau_{1}\right)} \exp \left(-\frac{t^{\prime}}{\tau_{1}}\right)\right.} \\
& \left.+\frac{2 c_{2}}{1+\exp \left(-a / \tau_{2}\right)} \exp \left(-\frac{t^{\prime}}{\tau_{2}}\right)\right] .
\end{aligned}
$$

Using the same terminology we have

$$
x\left(t^{\prime}\right)=\lim _{m \rightarrow \infty} u\left(t^{\prime}\right)=(-1)^{m}:
$$

By now it should be clear that the value $t_{0}^{\prime}$, obtained by solving

$$
r\left(t_{0}^{\prime}\right)=0
$$

with $a=n T$, is identical to the delay $t_{d}$ of Fig. 2. It is easy to verify that $r\left(t^{\prime}\right)$ is increasing through the zero determined by (25) when $m$ is even and decreasing when $m$ is odd, provided only that

$$
\tau_{0}<\max \left(\tau_{1}, \tau_{2}\right) \text {. }
$$

This completes the proof of (4).

\section{References}

[1] F. de Jager, "Delta modulation, a method of PCM transmission using the 1-unit code," Phillips Res. Rep., vol. 7, 1952, pp. 442-466. A. Tomozawa and H. Kaneko, "Companded delta modulation for COM-16, Feb. 1968, pp. 149-157

[3] P. P. Wang, "An absolute stability criterion for delta modulation." IEEE Trans. Commun. Technol. (Concise Papers), vol, COM-16, Feb. 1968 , pp. 186-188.

\section{Correspondence}

\section{Intelligible Crosstalk in Multiple-Carrier FM Systems with Amplitude Limiting and AM-PM Conversion}

Abstract-Intelligible crosstalk will occur in a multiple-carrier FM system when a gain slope versus frequency characteristic is followed by AM-PM conversion. This phenomenon can be particularly serious in a communication satellite repeater that utilizes a traveling-wave tube for output power amplification. A model that includes both amplitude saturation and AM-PM conversion is developed and the two-carrier intelligible crosstalk is calculated.

Correspondence approved by the Communication Theory Committee of the IEEE Communication Technology Group. Manuscript received December 7, 1970.

\section{INTRODUCTION}

The simultaneous transmission of frequency-division-multiplexed FM signals through a nonlinear system can result in intelligible crosstalk. In a voice-communication system this effect is particularly severe because it is manifested as intelligible whispering and can have a psychological impact considerably more severe than one might expect from the quantitative crosstalk level. In order for intelligible crosstalk to occur it is only necessary to have a frequency-dependent gain slope followed by AM-PM conversion [1]; however, amplitude limiting will affect the crosstalk magnitude.

The intelligible crosstalk level is determined in this analysis for a gain slope versus frequency characteristic followed by a 